\section{Social competence and adolescent psychosis}

SIR: Dalkin et al (BJP, February 1994, 164, 202-207) suggest that premorbid personality may shape the expression of symptoms in first-onset psychosis. Lack of social competence may be indicative of schizophrenic vulnerability, even in the absence of schizotypical symptoms or represent the earliest manifestation of illness (Strauss et al, 1974).

We performed a retrospective case study (Resch, 1992) of 74 patients with schizophrenic and schizoaffective disorders (diagnoses according to DSM-III-R; 33 males, 41 females; mean age 16 years), in which premorbid social competence was rated on the Premorbid Adjustment Scale (PAS; Cannon-Spoor et al, 1982). Premorbid adjustment was compared with clinical outcome after eight weeks of neuroleptic treatment. Criteria for clinical outcome were the same as those of Pearlson et al (1989) (complete remission, partial remission and no response). Raters of the PAS were blind to clinical outcome. Raters of clinical outcome were blind to information regarding premorbid adjustment. Statistical analysis was made by nonparametric analysis of variance. Patients were grouped by their diagnoses and their recovery.

Patients who showed complete remission after eight weeks had significantly lower PAS scores (good social function) during childhood than patients with poorer clinical outcome. The median PAS scores of the schizophrenic and schizoaffective patients with a good outcome were, respectively, 0.25 and 0.23 ; with partial remission 0.44 and 0.38 ; and no response $0.46(P<0.05)$ and 0.42 $(P<0.01)$

In early adolescence patients experiencing complete remission had the lowest median PAS scores: schizophrenic patients 0.43 , patients with schizoaffective psychosis 0.38 ; schizophrenic and schizoaffective patients with partial remission had median scores of 0.73 and 0.70 , respectively; and patients with no response to therapy $0.72(P<0.05)$ and 0.70 $(P<0.05)$.

Our study provides additional evidence that social competence is an essential aspect of the development of schizophrenia.

Poor premorbid social adjustment may be a prodromal sign of schizophrenia and prodromal signs may interfere with social adjustment during early adolescence. However, social competence in childhood is a feature of normal child development, before any prodromal signs may occur. Social competence in childhood is of prognostic relevance for the therapeutic outcome of psychosis in youth.
Cannon-Spoor, H. E., Porkin, S. G. \& Wyatt, R. J. (1982) Measurement of premorbid adjustment in chronic schizophrenia. Schizophrenia Bulletin, 8, 470-484.

Pearlson, G. D., Krieger, L., Rabins, P. V., et al (1989) A chart review study of late onset and early onset schizophrenia. American Journal of Psychiatry, 146, 1568-1574.

Resch, F. (1992) Therapie der Adoleszentenpsychosen. Stuttgart: Thieme-Copythek.

Strauss, J. S., Carpenter, W. \& BartKo, J. (1974) The diagnosis and understanding of schizophrenia. Speculations on the processes that underlie schizophrenic symptoms and signs. Schizophrenia Bulletin, 11, 61-69.

G. Paul Amminger

REgINA MUTSCHLECHNER

Universitätsklinik für Neuropsychiatrie des

Kindes- und Jugendalters

Vienna, Austria

Heidelberg, Germany

Franz Resch

\section{ICD-10: a neuropsychiatrist's nightmare?}

SIR: Lewis (BJP, February 1994, 164, 157-158) claims that five problems have been introduced with the term 'organic' in ICD-10 (World Health Organization, 1992). The author sees this as causing “a neuropsychiatrist's nightmare".

As individuals who took part in the drafting of Section F00-F09, "Organic, including symptomatic, mental disorders", we recognise the value of Lewis' critique and offer readers our comments on the five problems he presents.

(1) Lewis qualifies the ICD-10 explanatory note on the term 'organic' as confusing. We find the way he chooses to quote half a sentence out of context indeed confusing, and fail to see the alleged paradox if the two relevant sentences are read in full (we use italics for the parts of the paragraph omitted):

'Use of the term 'organic' does not imply that conditions elsewhere in this classification are 'nonorganic' in the sense of having no cerebral substrate. In the present context, the term 'organic' means simply that the syndrome so classified can be attributed to an independently diagnosable cerebral or systemic disease or disorder."

(2) He finds difficulty with the use of the terms 'symptomatic' and 'secondary' in ICD-10, saying that these are often tautologous and inconsistent. In our view, and that of many colleagues whom we consulted around the world, the introduction to the ICD-10 section on organic mental disorders (pp. 45-46) gives a particularly clear statement. It proposes that the organic mental disorders can be 